\title{
Analysis of protein interaction and function with a 3-dimensional MALDI-MS protein array
}

\author{
Igor M. Gavin, Alexander Kukhtin, David Glesne, Daniel Schabacker, and Darrell P. Chandler
}

BioTechniques 39:99-107(July 2005)

\begin{abstract}
Protein profiling and characterization of protein interactions in biological samples ultimately require indicator-free methods of signal detection, which likewise offer an opportunity to distinguish specific interactions from nonspecific protein binding. Here we describe a new 3-dimensional protein microchip for detecting biomolecular interactions with matrix-assisted laser desorption-ionization mass spectrometry (MALDI-MS); the microchip comprises a high-density array of methacrylate polymer elements containing immobilized proteins as capture molecules and directly interfaces with a commercially available mass spectrometer. We demonstrated the performance of the chip in three types of experiments by detecting antibody-antigen interactions, enzymatic activity, and enzyme-inhibitor interactions. MALDI-MS biochip-based tumor necrosis factor $\alpha(T N F-\alpha)$ immunoassays demonstrated the feasibility of detecting antigens in complex biological samples by identifying molecular masses of bound proteins even at high nonspecific protein binding. By detecting model interactions of trypsin with trypsin inhibitors, we showed that the protein binding capacity of methacrylate polymer elements and the sensitivity of MALDI-MS detection of proteins bound to these elements surpassed that of other 2- and 3-dimensional substrates tested. Immobilized trypsin retained functional (enzymatic) activity within the protein microchip and the specificity of macromolecular interactions even in complex biological samples. We believe that the underlying technology should therefore be extensible to whole-proteome protein expression profiling and interaction mapping.
\end{abstract}

\section{INTRODUCTION}

The discovery of protein biomarkers enables the early detection and accurate prognosis of many diseases (1). The ability to specifically detect and identify protein expression also helps determine the responses of an organism and/or host immune system to treatment. Many conventional methods of protein detection are based on the enzyme-linked immunosorbent assay (ELISA), measuring specific interaction between target proteins and antibodies immobilized on a solid support. However, conventional ELISA requires significant starting materials, so considerable research is directed toward miniaturizing ELISA-based techniques in the form of protein arrays (2-4). Antibody arrays detect changes in protein expression and, in this sense, are analogous to DNA arrays for gene expression analysis. Proteins are either labeled with fluorescent dye before applying to the chip or detected with a second antibody, specific to a different region of the molecule. Such ELISAtype sandwich immunoassays require a rigorous selection of a matched pair of capture and detection antibodies recognizing different parts of the protein with little or no spatial interference.

Similarly, drug discovery depends on designing effective inhibitors of protein function. Drug candidates have to be selected for their effect on the target protein in either activity or binding assays. Microarrays provide a high-throughput platform for screening drug candidates (5). However, affinity reagents used for signal detection in conventional arrays require a priori knowledge of interacting partners and therefore are not suitable for detecting interactions with uncharacterized, modified, or novel interacting molecules. Because affinity-based signal detection techniques cannot unambiguously distinguish specific interactions from nonspecifically bound proteins, the detection selectivity suffers from crossreactivity of affinity reagents, resulting in high background levels, which limits the number of individual reactions in one assay. Alternative methods to label or tag each sample may change its activity and biochemical properties $(6,7)$. Thus, detecting macromolecules and characterizing their interactions, espe- cially in complex mixtures (e.g., cell lysates, serum, and environmental samples), ultimately require indicator-free methods (8) that offer the potential to simultaneously detect uncharacterized molecules bound to the probe.

Mass spectrometry is capable of detecting many proteins simultaneously by identifying their molecular masses and does not necessarily require labeling or "tagging" analyte molecules. Several ionization methods such as fast atom bombardment (9), matrix-assisted laser desorption and ionization (MALDI) (10), and electrospray ionization (ESI) (11) are used as ion sources for mass spectrometry. MALDI is a wellestablished mass spectometry ionization technique and is accomplished by co-dispersing and crystallizing probes with a laser-absorbing matrix [e.g., sinapinic acid (SA), $\alpha$-cyano-4hydroxycinnamic acid $(\alpha-\mathrm{CHC}), 2,5-$ dihydroxybenzoic acid (DHB), etc.] on a sample plate. A key advantage of MALDI over ESI is the static nature of the ionization technique, a property that makes MALDI easily amenable to signal detection from microarray substrates (as opposed to the liquid 
probe introduction for ESI). A similar ionization technique is used by the surface-enhanced laser desorption/ionization time-of-flight mass spectrometry (SELDI-TOF-MS), a ProteinChip ${ }^{\circledR}$ system now sold by Ciphergen (Fremont, CA, USA) that is capable of detecting proteins bound to lowdensity, planar chromatographic, or protein arrays (12). MALDI-MS has also been combined with surface plasmon resonance technology (13). However, planar chip surfaces are known to denature proteins and induce steric constraints on binding and protein function $(14,15)$, and a 2-dimensional (2-D) surface has a characteristic (and relatively low) binding capacity that may affect lower detection limits and the linear dynamic range of the overall system.

Three-dimensional (3-D) gels were developed, in part, to overcome steric and probe density constraints imposed by 2-D surfaces while preserving the functional integrity of immobilized biomolecules (16-19). Recently, MALDI-MS has been used to demonstrate the feasibility of detecting proteins bound to 3-D acrylamide-based gel-pad arrays (20) as well as to continuous HydroGel ${ }^{\circledR}$ protein arrays (Perkin Elmer, Wellelsey, MA, USA) (21). A common criticism of gel substrates is the potentially restricted pore size of the matrix $(3,22)$ because small pores can limit diffusion and preclude large molecules from entering into the gel and forming complexes with the immobilized probe. To address the pore size constraints and improve the range of applications for 3-D microarrays, we are studying new polymers in which the porosity can be varied from tens to hundreds of nanometers. These new polymer matrices improve macromolecular diffusion in a mechanically, thermally, and chemically stable array element. The purpose of this study was therefore to develop a new 3-D MALDI-MS protein microchip for high-throughput detection, identification, and characterization of protein-protein interactions and activity in uncharacterized or complex biological backgrounds. The microchips incorporate a new 3-D element polymer that interfaces directly with a commercially available MALDI mass spectrometer.

\section{MATERIALS AND METHODS}

\section{Biochip Fabrication}

Photopolymerized methacrylate element arrays were manufactured as described by Yershov et al. (23), with the following modifications. A photopolymerization mixture of glycidyl methacrylate (GMA), ethylene dimethacrylate (EDMA), N-(5,6-di-o-isopropylidene) hexylacrylamide, and 2,2-dimethoxy2-phenyl-acetophenone (Aldrich, Milwaukee, WI, USA) was prepared by first mixing $20 \% \mathrm{~N}$-(5,6-di-o-isopropylidene) hexylacrylamide in GMA in a 6:4 (v/v) ratio with EDMA. The resulting solution was then mixed with cyclohexanol-dodecanol [9:1 (v/v)] in a ratio of $4: 6(\mathrm{v} / \mathrm{v})$. Three microliters of $10 \%(\mathrm{w} / \mathrm{v})$ 2,2-dimethoxy-2-phenylacetophenone in cyclohexanol were added to $100 \mu \mathrm{L}$ of the combined polymer solution, vortex mixed, and degassed for $3 \mathrm{~min}$. Next, a polymerization chamber was assembled consisting of either a no. $1 \frac{1 / 2}{2}$ rectangle microscope cover glass or glass microscope slide (Corning Life Sciences, Acton, MA, USA), treated with PlusOne ${ }^{\mathrm{TM}}$ BindSilane (Amersham Biosciences, Uppsala, Sweden), and a chromium-plated quartz mask containing transparent windows arranged in $200 \times 200-\mu \mathrm{m}$ squares on $2-\mathrm{mm}$ centers. The polymerization chamber was filled with the activated polymer mixture and exposed to a UV light from an Oriel UV Flood Exposure Source with $6 \times 6$ beam of light (Oriel Instruments, Stratford, CT, USA) powered by a $1000 \mathrm{~W} \mathrm{HgXe}$ lamp for $5 \mathrm{~min}$. After photopolymerization, the chamber was disassembled, and the polymerized 3-D array matrix was briefly rinsed with ethanol and water, washed in methanol for $30 \mathrm{~min}$ in methanol-water solution $[1: 1(\mathrm{v} / \mathrm{v})]$ for $30 \mathrm{~min}$, and then air-dried. The microchip matrices can be stored dried at room temperature for at least 1 year.

\section{Protein Immobilization}

Proteins were immobilized to the polymer backbone by covalently coupling protein amines with aldehyde groups in the polymer. Aldehyde groups were generated from GMA epoxide moieties in the array elements by incubating array polymer matrices in $0.5 \mathrm{M}$ boron trifluoride etherate in ethanol-water $[9: 1(\mathrm{v} / \mathrm{v})]$ for $3 \mathrm{~h}$. After washing with ethanol and water for $5 \mathrm{~min}$, the matrices were treated with $2 \%$ trifluoroacetic acid for $15 \mathrm{~min}$ and rinsed with water for $5 \mathrm{~min}$. Finally, the matrices were treated with $0.1 \mathrm{M}$ sodium periodate for $30 \mathrm{~min}$, rinsed with water, and dried.

Individual array elements were manually loaded with approximately $0.2 \mu \mathrm{L}$ of $100 \mu \mathrm{M}$ protein in immobilization buffer $(0.1 \mathrm{M} \mathrm{NaCl}, 10 \mathrm{mM}$ sodium phosphate, $\mathrm{pH} 7.0$, and $10 \%$ glycerol) corresponding to approximately $500 \times$ the 3 -D volume of an individual element. The chip was then incubated overnight in a sealed humid chamber containing a saturated solution of sodium chloride. Schiff bases (formed by the reaction of aldehyde groups in the polymer with protein amines) and remaining reactive aldehyde groups were reduced with $0.1 \mathrm{M}$ sodium borohydride for $5 \mathrm{~min}$, resulting in a stable covalent bond between the protein and the polymer matrix. Protein arrays were then rinsed once with Trisbuffered saline (TBS; Pierce, Rockford, IL, USA) for $10 \mathrm{~min}$, three consecutive washes with TBS plus $0.1 \%$ Tween ${ }^{\circledR}$ 20 (TBST) for $10 \mathrm{~min}$ each, and Superblock ${ }^{\mathrm{TM}}$ solution in TBS (Pierce) for $1 \mathrm{~h}$.

Protein immobilization on SuperAldehyde (Telechem International, Sunnyvale, CA, USA) and HydroGel substrates were carried out according to the manufacturers' recommendations. For direct comparisons between MALDI-MS arrays and the commercial substrates, the arrays were fabricated on a standard glass microscope slide, as described above, rather than on a glass coverslip.

\section{Interaction Assays and MALDI-MS Analysis}

Interaction assays between the protein arrays and target solutions were normally carried out in TBST in 20 or $200 \mu \mathrm{L}$ CoverWell ${ }^{\mathrm{TM}}$ incubation chambers (Grace Biolabs, Bend, OR, USA) for $2 \mathrm{~h}$ at room temperature. Unbound protein was removed by three consecutive washes with TBST for $10 \mathrm{~min}$ at room temperature; the array was brief- 
ly rinsed with water and air-dried. Two hundred nanoliters of MALDI matrix solution were then manually applied to each array element, and the array was air-dried and mounted directly onto a ground steel MALDI target MTP 384 TF (Bruker Daltonics, Billerica, MA, USA). The choice of MALDI matrix was determined in preliminary experiments where the application of $\alpha-\mathrm{CHC}$ resulted in higher signal intensity compared to other matrices, such as SA or DHB (data not shown). For protein arrays that were fabricated on commercially available substrates (coated glass microscope slides), we machined a 1-mm depression in an aluminum massive target MTP $384 \mathrm{~T}$ to maintain laser focus with the surface of the biochip. The plate was introduced into a Biflex $^{\mathrm{TM}}$ III mass spectrometer (Bruker Daltonics), and the mass spectrum from each array element was individually obtained according to the manufacturers' instructions. Normally, the signal was taken from 200 laser shots per array element from at least five replicate elements. Each experiment was repeated at least twice.

\section{MALDI-MS Analysis of On-Chip Protein Activity}

Trypsin was immobilized in MALDI-MS array elements as described above and incubated overnight in a sealed chamber at $37^{\circ} \mathrm{C}$ with $5 \mu \mathrm{L}$ of $10 \mu \mathrm{M}$ apomyoglobin (Sigma, St. Louis, MO, USA) in $10 \mathrm{mM}$ ammonium acetate, $\mathrm{pH}$ 6.0. Identical reactions were also performed in parallel with $1 \mu \mathrm{M}$ trypsin in standard $1.5-\mathrm{mL}$ polypropylene microcentrifuge tubes. Afterwards, 2 volumes of $\alpha$-CHC MALDI matrix solution were added to 1 volume of reaction mixture, $0.5 \mu \mathrm{L}$ of the mixture was transferred to the mass spectrometer target plate, dried, and analyzed with the mass spectrometer. Signal was taken from 200 laser shots per spot and from at least five spots on the MALDI plate. The experiment was repeated three times.

\section{Protein Interaction Assay in a Cell Lysate}

Human embryonic kidney (HEK)293 cells were cultured in plas- tic dishes (BD Biosciences, Franklin Lakes, NJ, USA) in Dulbecco's modified Eagle's medium (DMEM) medium supplemented with $10 \%$ heatinactivated fetal bovine serum (FBS; HyClone, Logan, UT, USA). The cells were incubated at $37^{\circ} \mathrm{C}$ in humidified air containing $8 \% \mathrm{CO}_{2}$. The cells were washed twice with ice-cold phosphatebuffered saline (PBS) and scraped from the bottom of the plate. The cells were collected by centrifugation at $500 \times g$ for $3 \mathrm{~min}$ and then incubated at $-20^{\circ} \mathrm{C}$ for $2 \mathrm{~h}$. An equal volume (normally $0.2-0.5 \mathrm{~mL}$ ) of homogenization buffer [10 mM Tris-HCl, pH 7.4, $250 \mathrm{mM}$ sucrose, 2 mM EDTA supplemented with the proteinase inhibitor cocktail, $0.1 \mathrm{mg} / \mathrm{mL} \quad \mathrm{N}$-p-tosyl-L-phenylalanine chloromethyl ketone (TPCK), 0.1 $\mathrm{mg} / \mathrm{mL}$ phenylmethylsulfonyl fluoride (PMSF), $1 \mu \mathrm{g} / \mathrm{mL}$ pepstatin, $1 \mu \mathrm{g} / \mathrm{mL}$ leupeptin, $2 \mu \mathrm{g} / \mathrm{mL}$ antipain] was added to the cell pellet, and the mixture was homogenized with a Dounce homogenizer (tight fit). The cell lysate was cleared by centrifugation at $100,000 \times g$ for $1 \mathrm{~h}$ and supplemented with trypsin (Sigma) at the indicated concentrations. The mixtures were then hybridized to the soybean trypsin inhibitor (STI; Sigma) immobilized on the chip, and the bound protein was detected with mass spectrometry as described above.

\section{On-Chip Immunoassays}

Anti-human IL-1 $\alpha$ monoclonal antibodies (Pierce) and mouse monoclonal anti-human TNF- $\alpha$ antibodies MAB1 (eBioscience, San Diego, CA, USA) were immobilized within array elements using $1 \mathrm{mg} / \mathrm{mL}$ stock solution. Protein arrays were then hybridized to either Texas Red-modified goat antimouse IgG (Molecular Probes, Eugene, OR, USA) or to RPMI 1640 medium supplemented with $10 \%$ heat-inactivated FBS (Harlan, Indianapolis, IN, USA), $100 \mu \mathrm{g} / \mathrm{mL}$ penicillin, $100 \mu \mathrm{g} / \mathrm{mL}$ streptomycin (Invitrogen, Rockville, MD, USA), and recombinant TNF- $\alpha$ (eBioscience) as described above.

\section{RESULTS}

\section{MALDI-MS Biochip Templates}

The MALDI-MS biochip template represents a high-density array of GMA polymer elements (typically $200 \times 200 \mu \mathrm{m})$ photopolymerized on a $0.17-\mathrm{mm}$ thin glass microscope coverslip in various patterns (the layout for manual loading is shown in Figure 1). Due to the smaller size of an individual element, the density of MALDI-MS chip elements is much higher than that of commercial SELDI or Biacore chips (Biacore AB, Uppsala, Sweden). Many biochip applications in our laboratory use other high-density layouts, accommodating thousands of elements on one chip. Probe loading to these chips is accomplished with an ANL custom-built, multipin robot (Quadrate II) specifically designed to load probes on preformed, three-dimensional gel element features without direct pin contact with the gel elements (see U.S. Patent nos. $5,851,776 ; 5,851,783$ and related patents). The ANL production facility has the capacity for 1000 biochips per month.

The hydrophobic separation between array elements prevents probe diffusion or mixing between test sites and allows repeated application of the probe without "spreading" and mixing probes. Our interest in methacrylate polymers stems from recent data showing that the methacrylate formulation increases protein binding capacity at least 5- to 7-fold compared to acrylamide substrates (A. Kukhtin, B. Chernov, J. Golova, G. Yershov, and M. Gemmell, unpublished data). Unlike acrylamide, methacrylate did not require hydration prior to protein immobilization, which provides a modest simplification

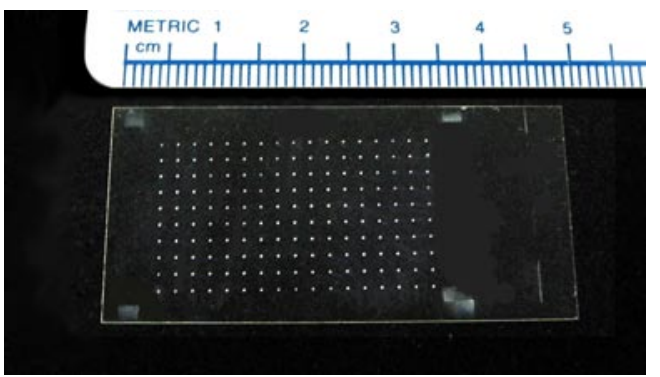

Figure 1. MALDI-MS protein biochip. The experimental chip for manual loading is shown. MALDI-MS, matrixassisted laser desorption-ionization mass spectrometry. 
of the biochip manufacturing process. Similary, preliminary tests indicate that methacrylate polymers are more rigid and thermally/chemically stable than acrylamide, facilitating their manipulation and analysis in biomolecular interaction assays. The biochip was easily mounted on a target plate of a commercial mass spectrometer, introduced into the instrument, and analyzed without refocusing the MALDI laser beam or recalibrating the instrument. The glass support made it possible to use the target plate's own coordinates for biochip positioning, but because the methacrylate polymer elements are visible, it is simple to locate individual array ele-
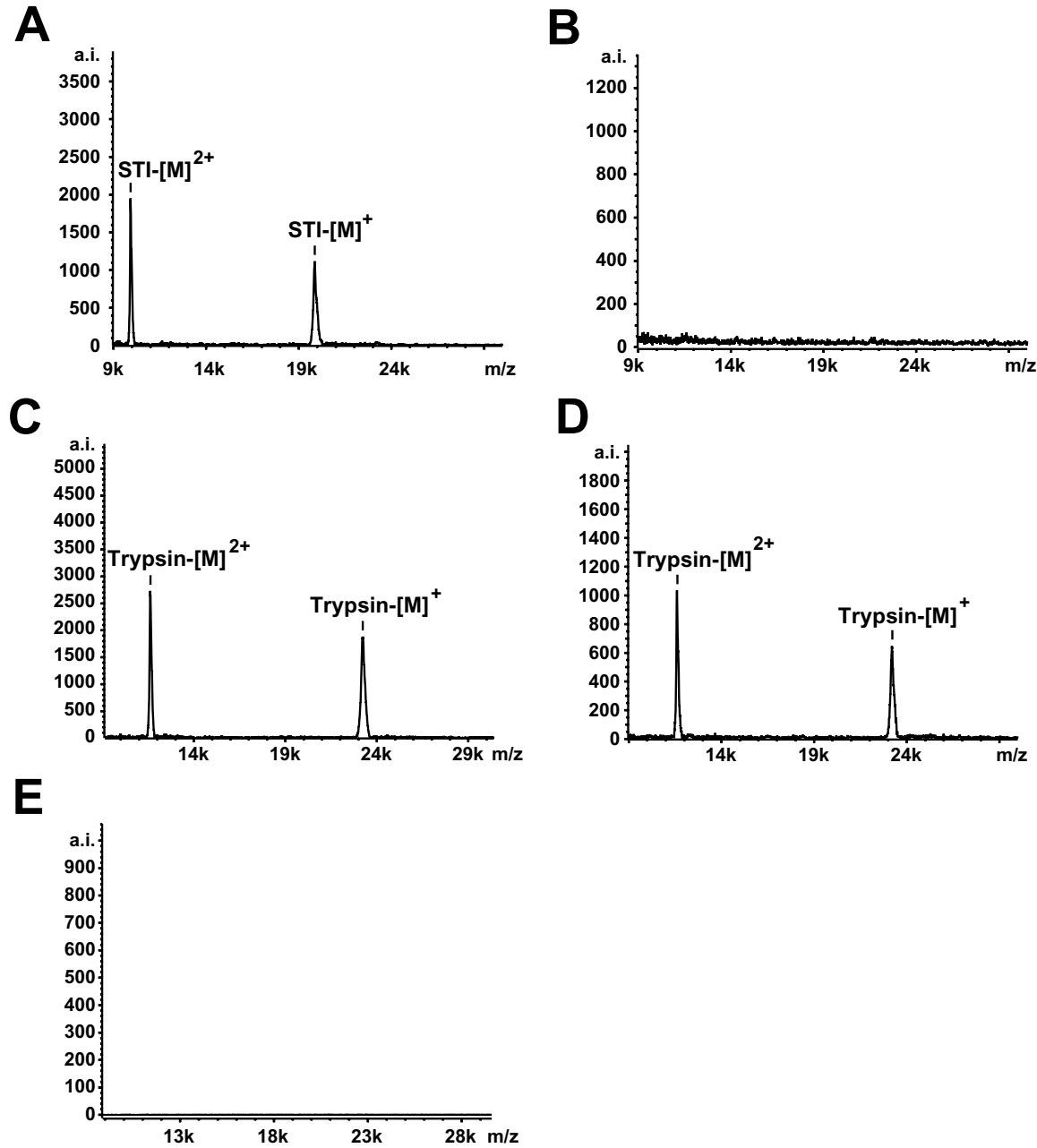

Figure 2. On-chip mass spectral analysis of trypsin interactions with trypsin inhibitor. Mass spectra of proteins bound to (A) chip-immobilized bovine trypsin, (B) BSA, (C) STI, (D) SPC1 trypsin inhibitor, or to (E) an empty element (i.e., negative control) are shown. The probes were immobilized in the array elements of the same chip by loading $10 \mathrm{mg} / \mathrm{mL}$ stock solutions to 5-10 array elements. Each chip was hybridized to either (A and B) $10 \mu \mathrm{g} / \mathrm{mL}$ STI or (C-E) $1 \mu \mathrm{g} / \mathrm{mL}$ trypsin. Each mass spectrum corresponds to 200 laser shots per element and is the representative signal from at least five elements in at least three hybrization experiments performed with different chips. BSA, bovine serum albumin; STI, soybean trypsin inhibitor; a.i., absolute intensity; $\mathrm{m} / \mathrm{z}$, mass-to-charge ratio. interaction between bovine pancreatic trypsin (BPT) and STI [association constant $K_{a}=10^{10} \mathrm{M}^{-1}$ (27)] regardless of which protein was immobilized in the array elements (Figure 2, A and C), indicating that specific functional interactions can occur within the methacrylate element array. To determine if the MALDI-MS arrays can be used to physically separate weakly interacting partners, we performed an identical experiment using the on-chip association of trypsin with SPC1 trypsin inhibitor $\left[\mathrm{K}_{\mathrm{a}}=10^{6} \mathrm{M}^{-1}(28)\right]$. Trypsin binding to immobilized inhibitor SPC1 was detected (Figure 2D), which shows that the MALDI-MS biochip system can also be used to interrogate lower affinity protein interactions. Control reactions between STI and immobilized bovine serum albumin (BSA; Figure 2B) and the application of MALDI matrix to a blank methacrylate element array incubated with trypsin (Figure 2E) resulted in no detectable signal, which suggests that the methacrylate polymer element formulation is relatively immune to nonspecific protein binding. The absence of any signal corresponding to the immobilized protein (Figure 2, $\mathrm{A}$ and $\mathrm{C}$ ) demonstrates that polymerimmobilized protein or capture probe is not released from the array element during laser desorption and ionization.

Detecting on-chip protein interactions is not equivalent to detecting or characterizing protein function. Indeed, covalently attaching proteins to a surface often results in a loss of biological activity $(3,4,22,29)$. To determine if trypsin immobilization to the methacrylate polymer also retains protein activity, trypsin was immobilized in methacrylate element arrays and incubated with $10 \mu \mathrm{M}$ apomyoglobin overnight at $37^{\circ} \mathrm{C}$. As shown in Figure 3, the spectrum of products of apomyoglobin proteolysis by immobilized trypsin was similar to the spectrum obtained from a test-tube control reaction even though lower signal was detected in the case of the on-chip reaction. However, the absence of partial digestion products in the spectra suggests that the proteolysis was completed in both samples and indicates that protein immobilization within the methacrylate polymer element did not destroy enzyme function and activity. 
To determine if methacrylate array elements could be used to spatially isolate interacting partners in cell extracts, various concentrations of STI were added to HEK293 cell lysates and hybridized to polymer-immobilized bovine trypsin. As shown in Figure 4, the prototype MALDI-MS biochip system can specifically detect as little as $1.5 \mathrm{pM}$ of total STI amount in the cell lysate. These data demonstrate that the methacrylate polymer element array can be utilized as an affinity purification platform for MALDI-MS analysis of proteins and interacting partners in biological samples of various complexities.

\section{Biochip Substrates}

MALDI-MS-based detection techniques can certainly be used in combination with planar $(12,13)$ and continuous HydroGel (21) substrates. We hypothesized that discrete methacrylate polymer elements will provide higher probe immobilization capacity and, thus, improved detection limits and/or dynamic range than other substrates. To begin addressing this hypothesis, we performed a comparison between commercially available microarray substrates and the methacrylate element arrays developed and described here. TeleChem International's SuperAldehyde slides were utilized as a template for planar arrays because the protein immobilization chemistry is similar to the one used for the methacrylate polymer described here. Perkin Elmer's 3-D acrylamide-based HydroGel has been shown to be conducive to on-chip MALDI-MS analysis (21) and represents a commercially available 3-D surface. Because both SuperAldehyde and HydroGel surface chemistries are deposited on glass microscope slides, we likewise manufactured the methacrylate element arrays on glass slides for these experiments.

STI was immobilized at various concentrations, and the respective arrays were hybridized to trypsin solution. As shown in Figure 5A, the planar substrate did not produce any detectable signal even at the highest concentration of immobilized capture protein. These results suggest that the planar surface is either denaturing trypsin, orienting trypsin in a sterically unfavorable manner for interaction, providing limited probe binding capacity, or any combination thereof. At the same time, both the continuous HydroGel (Figure 5B) and methacrylate element (Figure 5C) arrays generated detectable signal. However, the methacrylate elements generated more intense signal than continuous HydroGel. The improved signal of methacrylate elements relative to continuous HydroGel suggests that the methacrylate elements are providing a higher probe-loading capacity (thus improved capacity for interaction) than continuous HydroGel or that the methacrylate polymer formulation provides better binding conditions than acrylamide. Both interpretations are consistent with higher immobilization capacity and detection limits of methacrylate element-based antibody arrays measured with fluorescent detection

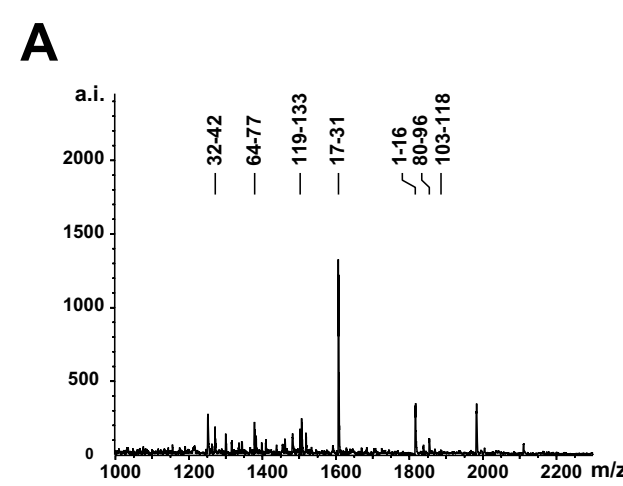

B

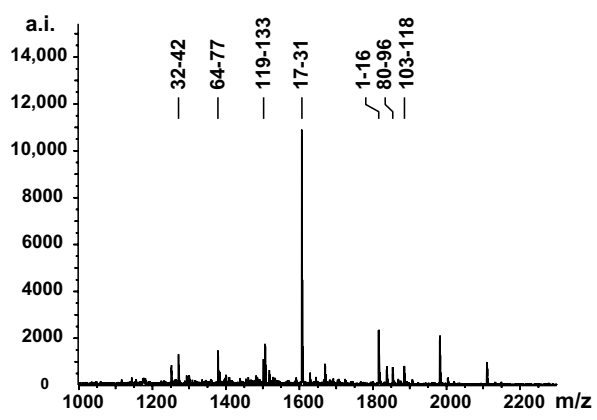

Figure 3. Proteolytic activity of immobilized trypsin. The mass spectra of products of apomyoglobin digestion with (A) chip-immobilized trypsin and with (B) trypsin solution are shown. Note that peptide distribution is similar in both spectra, and all expected peptide fragments shown on the top of both panels are present. For other details, see Materials and Methods. a.i., absolute intensity; $\mathrm{m} / \mathrm{z}$, mass-to-charge ratio. techniques (A. Kukhtin, B. Chernov, J. Golova, G. Yershov, and M. Gemmell, unpublished data).

\section{On-Chip Immunoassays}

As discussed earlier, selective labeling of analyte molecules may not be possible in clinical or environmental samples; therefore, many protein array platforms use affinity reagents such as antibodies or aptamers to detect bound proteins. Many of these molecules are cross-reactive, and their mixing in one assay increases nonspecific binding, which in turn decreases the selectivity of protein detection. At the same time, mass spectrometry can easily discriminate the target protein from other proteins bound to the same array element. Application of the MALDI-MS-based signal readout in protein expression profiling would eliminate the need for detection antibodies, thus a large number of target molecules can be detected in one assay. To explore the extensibility of methacrylate biochips to im-

$500 \mathrm{nM}$ STI
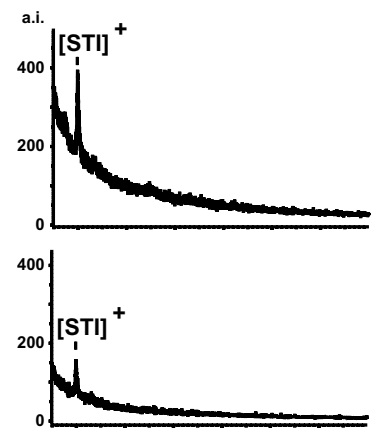

$50 \mathrm{nM} \mathrm{STI}$
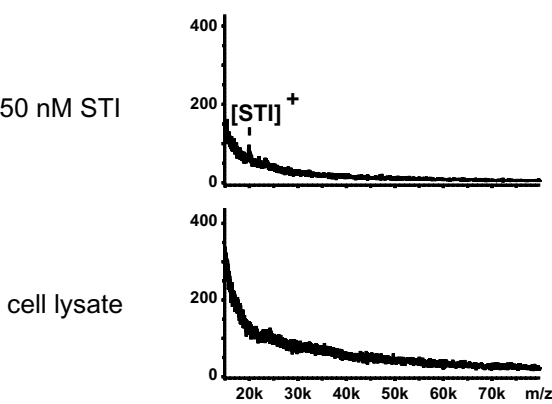

Figure 4. Detection of trypsin-STI interactions in cell lysates. Mass spectra of STI hybridized to trypsin chip in the cell lysate are shown. The HEK293 cell lysate corresponding to $4 \times 10^{7}$ cells/ $\mathrm{mL}$ was supplemented with STI at concentrations shown on the left of each panel and was hybridized to the chip-immobilized trypsin as described in Materials and Methods. For other details, see the legend to Figure 1. STI, soybean trypsin inhibitor; HEK, human embryonic kidney. a.i., absolute intensity; $\mathrm{m} / \mathrm{z}$, mass-to-charge ratio. 


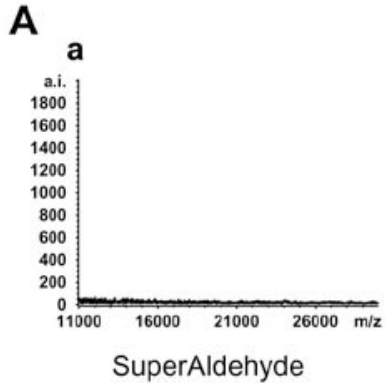

B

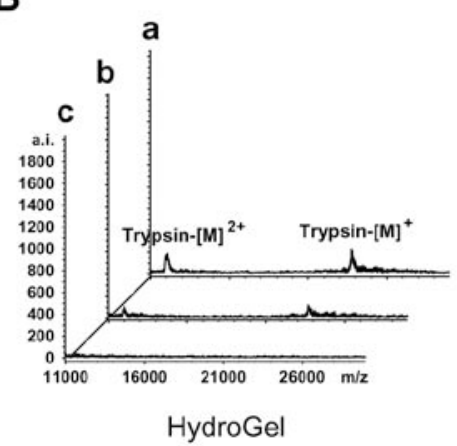

C

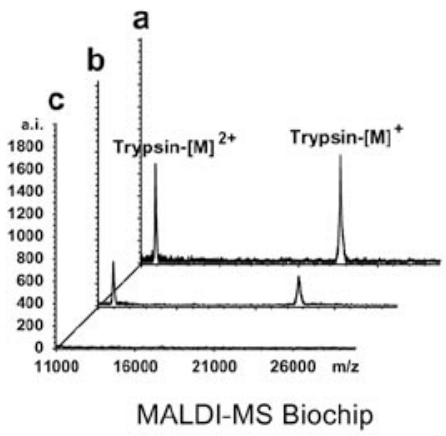

Figure 5. Protein binding capacity of three microarray substrates. The mass spectra of trypsin bound to chip-immobilized STI are shown. STI was immobilized on a (A) SuperAldehyde, (B) HydroGel, or (C) methacrylate slide at concentrations of (a) $10 \mathrm{mg} / \mathrm{mL}$, (b) $1 \mathrm{mg} / \mathrm{mL}$, or (c) $0.1 \mathrm{mg} / \mathrm{mL}$. Slides were hybridized to $1 \mu \mathrm{g} / \mathrm{mL}$ trypsin solution. For other details, see the legend to Figure 1. a.i., absolute intensity.

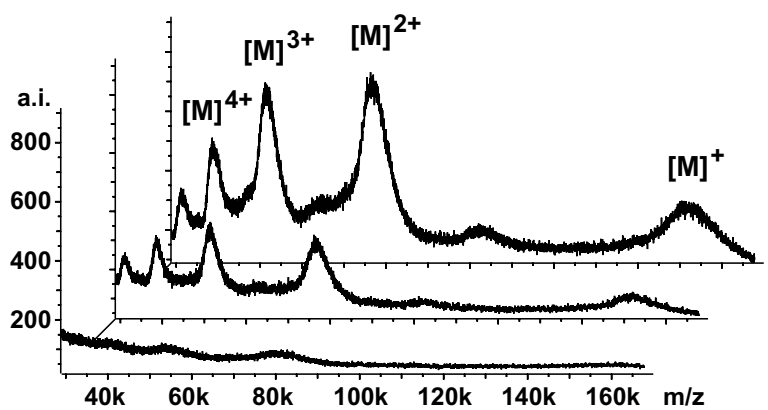

anti-mouse IgG

$700 \mathrm{nM}$

$70 \mathrm{nM}$

$7 \mathrm{nM}$

Figure 6. MALDI-MS chip-based immunoassay. Mass spectra of Texas Red-modified goat anti-mouse IgG bound to chip-immobilized mouse anti-human IL-1 $\alpha$ antibody are shown. Goat IgG concentration in hybridization buffer is shown on the right of each spectrum. For other details, see the legend to Figure 1. MALDI-MS, matrix-assisted laser desorption-ionization mass spectrometry; IgG, immunoglobulin G; IL-1 $\alpha$, interleukin- $1 \alpha$; a.i., absolute intensity; $\mathrm{m} / \mathrm{z}$, mass-to-charge ratio. methacrylate, acrylamide (16), and HydroGel biochips in this type of assay. The anti-human TNF- $\alpha$ monoclonal capture antibody MAB1 was immobilized on the slides and hybridized to unpurified cell culture medium containing human recombinant TNF- $\alpha$ at various concentrations. As shown in Figure 7 , a concentration-dependent peak corresponding to the secreted form of TNF- $\alpha$ is observed at concentrations higher than $1 \mathrm{ng} / \mathrm{mL}$ for methacrylate and acrylamide arrays (Figure 7, A and $\mathrm{B}$ ) and higher than $10 \mathrm{ng} / \mathrm{mL}$ for HydroGel slides (Figure7C). Methacrylate biochips detected as little as $20 \mathrm{fM}$ of total TNF- $\alpha$ amount in $10^{7}$ excess of cell culture medium proteins $(\mathrm{w} / \mathrm{w})$ and generated the highest signal among the microarray substrates, even amid nonspecific interactions between proteins present in the cell medium and the array elements. These data correlate with the increased protein binding capacity of methacrylate chips compared to other platforms observed with mass spectrometry (Figure 5) and optical signal detection (A. Kukhtin, B. Chernov, J. Golova, G. Yershov, and M. Gemmell, unpublished data). These experiments also demonstrate the feasibility of using methacrylate MALDI-MS biochips for antibody-based protein expression profiling. Several opportunities for increasing MALDI-MS biochip detection limits and decreasing nonspecific binding to methacrylate polymer elements are available and are the topic of ongoing research.

\section{DISCUSSION}

In this study, we describe the use of a new methacrylate polymer for fabricating 3-D element protein arrays and demonstrate the utility of methacrylate element protein arrays as an affinity separation chip for MALDI-MS analysis of protein interactions. Mass spectrometry clearly offers the opportunity to detect proteins and identify their molecular masses without a priori knowledge of protein expression or generating affinity reagents for detecting each target of interest. SELDI-MS, in particular, is a well-known, commercially available platform for chromatographic separations and protein detection on 

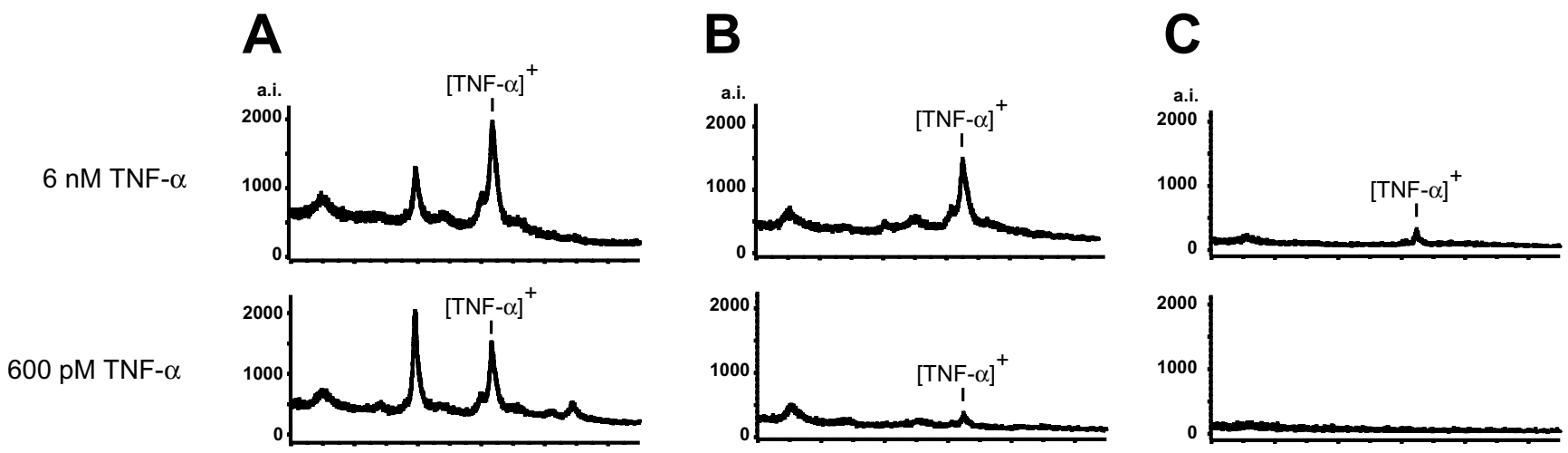

60 pM TNF- $\alpha$
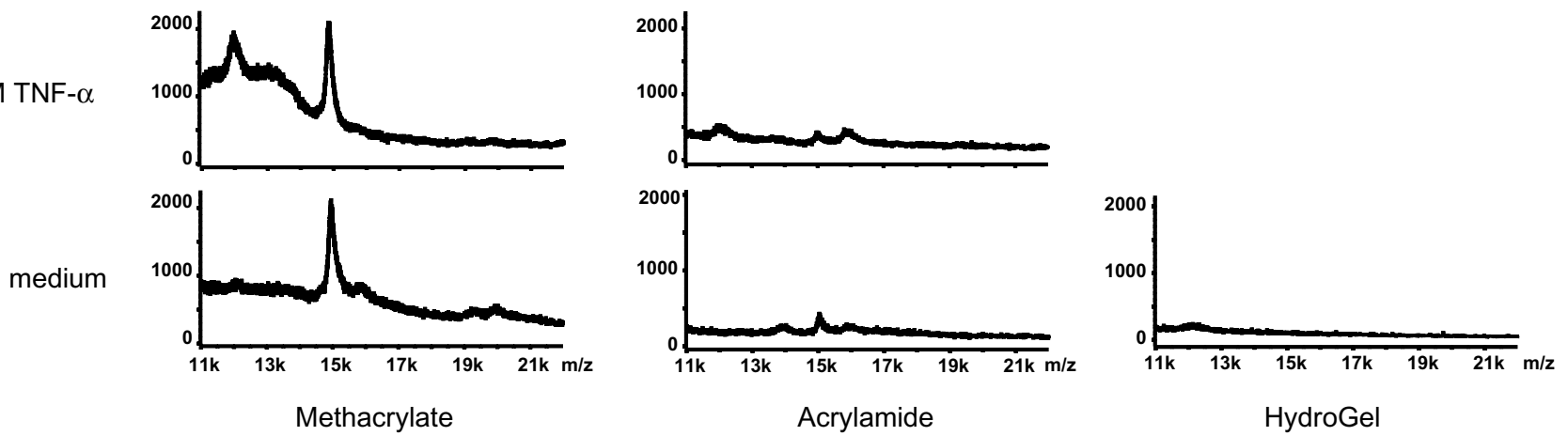

Figure 7. TNF- $\alpha$ detection in cell culture medium by three 3-D biochip substrates. The mass spectra of proteins bound to chip-immobilized anti-human TNF- $\alpha$ antibody MAB1 are shown. MAB1 was immobilized on three different 3-D biochip substrates: (A) methacrylate, (B) acrylamide, and (C) HydroGel, following hybridization to the cell culture medium supplemented with the recombinant TNF- $\alpha$ at concentrations shown on the left of each panel. For other details, see the legend to Figure 1. TNF- $\alpha$, tumor necrosis factor $\alpha$; a.i., absolute intensity; m/z, mass-to-charge ratio.

2-D surfaces, but interaction assays on planar substrates are subject to the limitations of 2-D surfaces, as previously described in detail (14) and illustrated in Figure 5A. At the same time, the current SELDI platform offers limited multiplexing capacity in a homogenous assay (six spots per a chip), a potentially significant limitation for deciphering molecular networks and complexes at the whole-proteome scale. Moreover, the relatively high (capital and operating) expense of commercial systems precludes their use by investigators who do not run binding assays on a regular basis. The 3-D element microchips, on the other hand, can be manufactured in-house at a relatively low cost and can be interfaced with virtually any offthe-shelf mass spectrometer without modifying the instrument.

The promise and potential of 3-D biochip substrates is that they will overcome the steric and probe immobilization capacity constraints of planar biochips, while preserving biomolecular integrity, function, and activity. Early studies demonstrated the feasibility of using mass spectrometry to detect hybridization signal from acrylamide- based protein chips (20,21). As shown here (Figures 5 and 7), however, planar glass, acrylamide, and HydroGel substrates have a lower MS-based detection sensitivity (and/or protein binding capacity) than methacrylate, a potential limitation for detecting weak interactions. The data further show that a 3-D methacrylate polymer element array preserves the functional integrity and biochemical activity of immobilized biomolecules (Figure 3), providing a clear path for developing comprehensive protein arrays for functional interaction and activity mapping as opposed to simply detecting a (specific or nonspecific) binding event.

Applying the MALDI-MS protein array technology to multiplex protein profiling in biological fluids may also alleviate many drawbacks of the conventional antibody arrays using optical detectors. That is, a multiplexed antibody or ELISA array assumes that all interactions between target and cognate antibody are absolutely specific, an assumption that may or may not be valid $(6,7)$. As illustrated in Figures 4 and 7 , the identification of molecular masses of spatially captured proteins from complex mixtures (e.g., cell lysates, serum, and environmental samples) will eliminate the need for specific detection antibodies and will allow protein expression analysis to be carried out with virtually an unlimited number of probes regardless of a priori knowledge or occurrence of interacting partners. Although less sensitive than conventional ELISA, the MALDI-MS biochip analysis did read through the complex background and any nonspecific binding of molecules in the TNF- $\alpha$ assay (Figure 7), which affords the opportunity to unambiguously detect proteins specifically interacting with each of the immobilized capture probes by identifying their molecular masses. From this perspective, relatively nonspecific antibodies, capture probes, and/or interacting partners can therefore be used in principle as capture molecules in methacrylate polymer element arrays for affinity-based separations prior to mass spectrometry detection, a capability that will facilitate the development and analysis of whole-proteome chips (15).

On-chip signal detection by MALDI-MS clearly offers an oppor- 
tunity to obtain sequence information on proteins by identifying products of proteolysis of analyzed proteins with sequence-specific proteases or by chemical cleavage (31). Proteins can be identified either by sequencing corresponding peptides with tandem mass spectrometry or by matching peptide masses with masses of predicted products of proteolysis of known proteins in the database (peptide fingerprinting). Further development of the mass spectrometry biochip as a high-throughput platform for the identification of molecules interacting with proteins of interest will facilitate the functional characterization of complete proteomes and the understanding of the role of every protein in the cell.

\section{ACKNOWLEDGMENTS}

This research was supported by the U.S. Department of Energy, Office of Science, under contract no. W-31-109ENG-38.

\section{COMPETING INTERESTS STATEMENT}

The authors declare no competing interests.

\section{REFERENCES}

1.Petricoin, E.F. and L.A. Liotta. 2002. Proteomic analysis at the bedside: early detection of cancer. Trends Biotechnol. 20:S30-S34.

2.Templin, M.F., D. Stoll, M. Schrenk, P.C. Traub, C.F. Vohringer, and T.O. Joos. 2002. Protein microarray technology. Trends Biotechnol. 20:160-166.

3.Zhu, H. and M. Snyder. 2003. Protein chip technology. Curr. Opin. Chem. Biol. 7:55-63.

4.Kusnezow, W. and J.D. Hoheisel. 2002. Antibody microarrays: promises and problems. BioTechniques 33(Suppl):14-23.

5.Ryan, T.E. and S.D. Patterson. 2002. Proteomics: drug target discovery on an industrial scale. Trends Biotechnol. 20:S45-S51.

6.Smith, R.D., L. Pasa-Tolic, M.S. Lipton, P.K. Jensen, G.A. Anderson, Y. Shen, T.P. Conrads, H.R. Udseth, et al. 2001. Rapid quantitative measurements of proteomes by Fourier transform ion cyclotron resonance mass spectrometry. Electrophoresis 22:16521668.

7.MacBeath, G. 2002. Protein microarrays and proteomics. Nat. Genet. 32(Suppl):526-532.

8.James, P. 2002. Chips for proteomics: a new tool or just hype? BioTechniques 33(Suppl):4-
13.

9.Barber, M., R.S. Bordoli, R.D. Sedgwick, and A.N. Tyler. 1981. Fast atom bombardment of solids as an ion source in mass spectrometry. Nature 293:270-275.

10.Karas, M. and F. Hillenkamp. 1988. Laser desorption ionization of proteins with molecular masses exceeding 10,000 daltons. Anal. Chem. 60:2299-2301.

11.Fenn, J.B., M. Mann, C.K. Meng, S.F. Wong, and C.M. Whitehouse. 1989. Electrospray ionization for mass spectrometry of large biomolecules. Science 246:64-71.

12.Merchant, M. and S.R. Weinberger. 2000. Recent advancements in surface-enhanced laser desorption/ionization-time of flight-mass spectrometry. Electrophoresis 21:1164-1177.

13.Nedelkov, D. and R.W. Nelson. 2003. Surface plasmon resonance mass spectrometry: recent progress and outlooks. Trends Biotechnol. 21:301-305.

14.Schaeferling, M., S. Schiller, H. Paul, M. Kruschina, P. Pavlickova, M. Meerkamp, C. Giammasi, and D. Kambhampati. 2002. Application of self-assembly techniques in the design of biocompatible protein microarray surfaces. Electrophoresis 23:3097-3105.

15.Zhu, H., M. Bilgin, R. Bangham, D. Hall, A. Casamayor, P. Bertone, N. Lan, R. Jansen, et al. 2001. Global analysis of protein activities using proteome chips. Science 293:21012105.

16.Arenkov, P., A. Kukhtin, A. Gemmell, S. Voloshchuk, V. Chupeeva, and A. Mirzabekov. 2000. Protein microchips: use for immunoassay and enzymatic reactions. Anal. Biochem. 278:123-131.

17.Mirzabekov, A. and A. Kolchinsky. 2002. Emerging array-based technologies in proteomics. Curr. Opin. Chem. Biol. 6:70-75.

18.Angenendt, P., J. Glokler, D. Murphy, H. Lehrach, and D.J. Cahill. 2002. Toward optimized antibody microarrays: a comparison of current microarray support materials. Anal. Biochem. 309:253-260.

19.Wang, C.C., R.P. Huang, M. Sommer, H. Lisoukov, R. Huang, Y. Lin, T. Miller, and J. Burke. 2002. Array-based multiplexed screening and quantitation of human cytokines and chemokines. J. Proteome Res. 1:337-343.

20.Rubina, A.Y., E.I. Dementieva, A.A. Stomakhin, E.L. Darii, S.V. Pan'kov, V.E. Barsky, S.M. Ivanov, E.V. Konovalova, et al. 2003. Hydrogel-based protein microchips: manufacturing, properties, and applications. BioTechniques 34:1008-1022.

21.Scrivener, E., R. Barry, A. Platt, R. Calvert, G. Masih, P. Hextall, M. Soloviev, and J. Terrett. 2003. Peptidomics: a new approach to affinity protein microarrays. Proteomics 3:122-128

22.Cutler, P. 2003. Protein arrays: the current state-of-the-art. Proteomics 3:3-18.

23.Yershov, G., V. Barsky, A. Belgovskiy, E. Kirillov, E. Kreindlin, I. Ivanov, S. Parinov, D. Guschin, et al. 1996. DNA analysis and diagnostics on oligonucleotide microchips. Proc. Natl. Acad. Sci. USA 93:4913-4918.

24.Zhu, G., Q. Huang, Y. Zhu, Y. Li, C. Chi, and Y. Tang. 2001. X-Ray study on an artificial mung bean inhibitor complex with bovine beta-trypsin in neat cyclohexane. Biochim.
Biophys. Acta 1546:98-106.

25.Helland, R., G.I. Berglund, J. Otlewski, W. Apostoluk, O.A. Andersen, N.P. Willassen, and A.O. Smalas. 1999. High-resolution structures of three new trypsin-squash-inhibitor complexes: a detailed comparison with other trypsins and their complexes. Acta Crystallogr. D Biol. Crystallogr. 55(Pt 1):139-148.

26.Song, H.K. and S.W. Suh. 1998. Kunitz-type soybean trypsin inhibitor revisited: refined structure of its complex with porcine trypsin reveals an insight into the interaction between a homologous inhibitor from Erythrina caffra and tissue-type plasminogen activator. J. Mol Biol. 275:347-363.

27.Brandts, J.F. and L.N. Lin. 1990. Study of strong to ultratight protein interactions using differential scanning calorimetry. Biochemistry 29:6927-6940.

28.Li, Y., Q. Huang, S. Zhang, S. Liu, C. Chi, and Y. Tang. 1994. Studies on an artificial trypsin inhibitor peptide derived from the mung bean trypsin inhibitor: chemical synthesis, refolding, and crystallographic analysis of its complex with trypsin. J. Biochem. (Tokyo) 116:18-25

29.Elia, G., M. Silacci, S. Scheurer, J. Scheuermann, and D. Neri. 2002. Affinity-capture reagents for protein arrays. Trends Biotechnol. 20:S19-S22.

30.Huang, R.P. 2004. Cytokine protein arrays. Methods Mol. Biol. 278:215-232.

31.James, P. 2001. Mass spectrometry and the proteome, p. 1-9. In P. James (Ed.), Proteome Research: Mass Spectrometry. Springer-Verlag, New York.

Received 5 November 2004; accepted 13 February 2005.

Address correspondence to:

Igor M. Gavin

Biochip Technology Center, Argonne National

Laboratory

9700 S. Cass Ave.

Argonne, IL 60439, USA

e-mail:igavin@anl.gov

To purchase reprints

of this article, contact

apfeffer@BioTechniques.com 